prototype of the new switching power amplifier and the later improvements.

\section{REFERENCES}

[1] Slobodan Cuk and R. D. Middlebrook, "A new optimum topology switching dc-to-dc converter," in 1977 IEEE Power Elec. Specialists Conf. Rec., pp. 160-179.

[2] Slobodan Cuk, "Modelling, analysis, and design of switching converters," Ph.D. thesis, California Inst. Technology, Nov. 1976. Also, NASA Rep. CR-135174.

[3] Slobodan Cuk and R. D. Middlebrook, "Coupled-inductor and other extensions of a new optimum topology switching dc-to-dc converter," in 1977 IEEE Industry Applications Soc. Ann. Meet. Rec., pp. 1110-1126.

[4] R. D. Middlebrook and Slobodan Ćuk, "Isolation and multiple output extensions of a new optimum topology switching dc-to-dc converter," in 1978 IEEE Power Electronics Specialists Conf. Rec., pp. 256-264.

[5] R. D. Middlebrook, Slobodan Cuk, and W. Behen, "A new battery charger/discharger converter," in 1978 IEEE Power Electronics Specialists Conf. Rec., pp. 251-255.

[6] L. Shaeffer, "VMOS-A breakthrough in power MOSFET technology," Siliconix Applications Note AN76-3, Santa Clara, CA 1976.

[7] R. D. Middlebrook and Slobodan Cuk, "Modelling and analysis methods for dc-to-dc switching converters,"' (invited review paper) in 1977 IEEE Int. Semiconductor Power Converter Conf. Rec., pp. 90-111.

[8] Slobodan Cuk and Robert W. Erickson, "A conceptually new highfrequency switched-mode amplifier technique eliminates current ripple,"' in Proc. Fifth Nat. Solid-State Power Conversion Conf., May 1978, pp. G3.1-G3.22.

\title{
Advances in Switched-Mode Power Conversion Part II
}

\author{
SLOBODAN ĆUK, MEMBER, IEEE, AND R. D. MIDDLEBROOK, FELLOW, IEEE
}

\begin{abstract}
A number of important practical extensions to the basic Cuk converter are presented. They include dc isolation, multipleoutput power sources, and a physical realization of the sought for hypothetical dc-to-dc transformer, a device which converts from pure dc (no voltage or current ripple) at one terminal, to pure dc (at a different voltage) at the other terminal. The application of the circuit in a highly efficient amplifier for the servo control of a de motor or other loads is also presented.
\end{abstract}

\section{INTRODUCTION}

$\mathbf{I}_{\mathrm{t} y}^{\mathrm{N}}$ PART I of this series we provided a review of the basic types of switched-mode power converters and showed how the effort to solve the characteristic problems of these earlier designs led to the development of a fundamentally new converter configuration. Fig. 1 shows a summary of the basic converter types and a physical realization of the new converter in its simplest form. The new converter topology embodies all of the desirable features of previous types while retaining none of their liabilities. Whereas other converters rely upon the inductive coupling of energy between the input and output, the new converter uses a capacitor to transfer stored energy between input and output inductors as shown.

Due to the presence of inductors on both the input and output, the current on either terminal remains continuous

Manuscript received October 15, 1982.

The authors are with the California Institute of Technology, Pasadena, CA 91125.

This article is reprinted from Robotics Age Magazine. avoiding the electrical noise problems associated with switching either the input or output current and resulting in increased conversion efficiency. The new converter has the desirable property that its output voltage can be either higher or lower than that of the input supply, as determined by the duty ratio of the switching transistor (the fraction $D$ of the switching period $\left(T_{s}\right)$ that the transistor is turned on).

Also presented in Part I were several important extensions to the basic converter design. These include adding the capability for bidirectional power flow between input and output, coupling the input and output inductors via a single transformer core, and a high-performance switched-mode power amplifier configuration using parallel converters driving a differentially connected load.

An important result of the coupled-inductor extension of the new converter, apart from the further reduction in the number of components, is that, by proper adjustment of the magnetic coupling between the input and the output inductors, the residual current and ripple at one of the terminals can be reduced exactly to zero, resulting in pure dc. Naturally, this motivated the search for a converter configuration which would achieve the desired characteristic of having zero current ripple at both input and output simultaneously, thus resulting in a physical realization of an ideal dc-to-dc "transformer." (See Fig. 8 in Part I.)

In this article, we will show how, by pursuing the desirable property of $\mathrm{dc}$ isolation between the input and the output cir- 
BUCK POWER STAGE

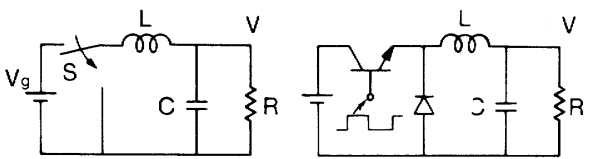

(a)

BOOST POWER STAGE

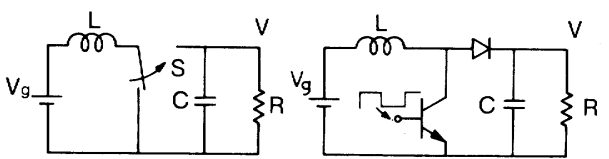

(b)

BUCK- BOOST POWER STAGE

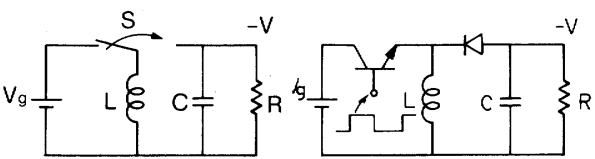

(c)

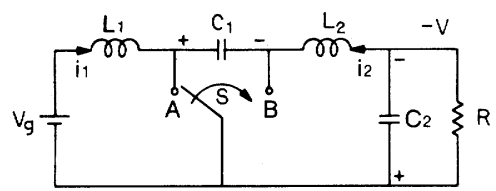

(d)

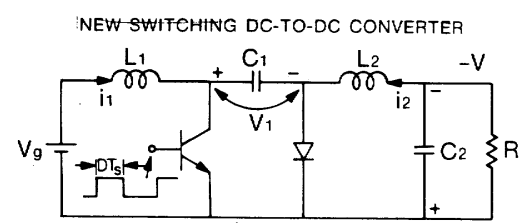

(e)

Fig. 1. (a)-(d) The family of four basic switching converter topologies and (e) a practical implementation of the Cuk converter using a transistor-diode combination. (a) Buck power stage. (b) Boost power stage. (c) Buck-boost power stage. (d) New converter topology. (e) New switching dc-to-dc converter.

cuits, the sought for solution for the ideal converter was found inherently derived from the new converter topology. Since the new topology provides higher performance than all other converter types, but may be implemented using fewer components than comparable solutions, it may be said to be an optimal design. To illustrate this point, we will present the design of a working circuit that shows the ease of employing the new topology in practical applications.

\section{DC ISOLATION IN THE NEW SWITCHING CONVERTER}

All the basic switching dc-to-dc converter-types reviewed in Part I, along with the newly introduced converter topology, are distinguished by the elegant method by which conversion is accomplished through the operation of a single switch (double-pole, single-throw) in an otherwise passive energy storage network, as shown in the converters in Fig. 1.

Despite this simple structure, which results in the high conversion efficiency characteristic of "switchers," the basic converter configurations all lack one of the properties often required of power suppliers: dc isolation between the input and output ports. There is therefore a strong incentive to find a
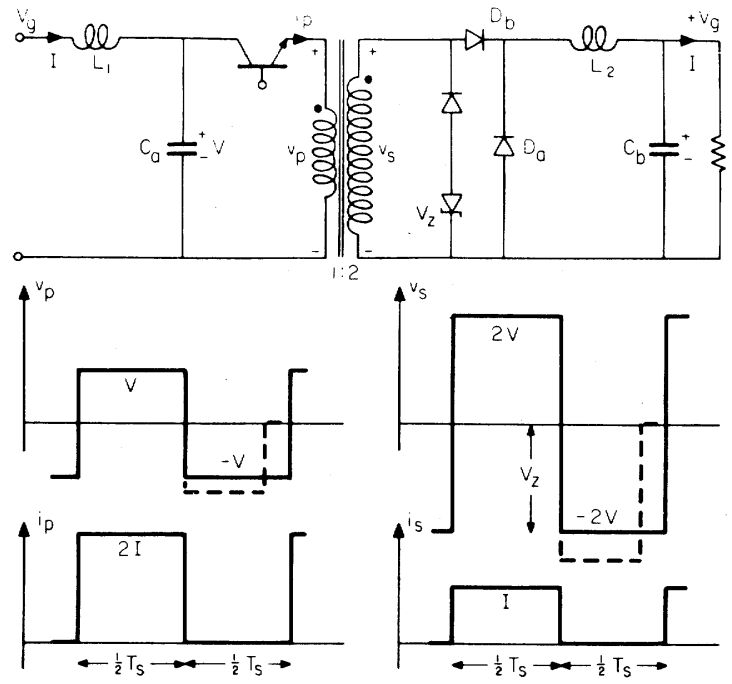

(a)
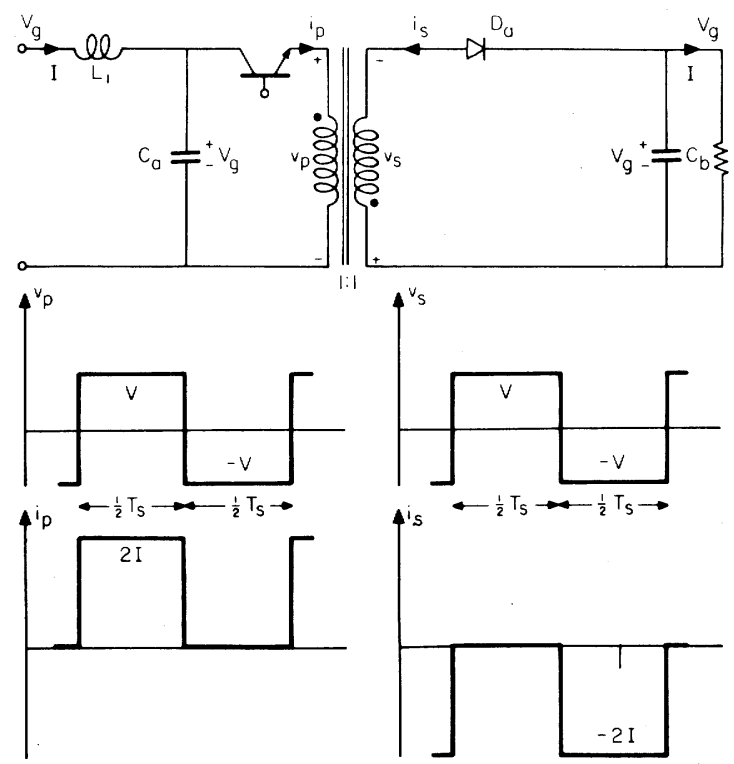

(b)

Fig. 2. Isolated versions of the (a) basic buck ("forward") and (b) buck-boost ("flyback") switching converters.

way to introduce an isolation transformer into the new converter design. One could of course, immediately proceed in a conventional manner and try a "push-pull" configuration using an isolation transformer with a center-tapped primary. However, the consequent doubling of the number of components, along with other practical problems inherent in such a design, favor the search for a less complex single-switch implementation using a "single-ended" isolation transformer.

For example, dc isolation can be introduced into the basic "buck" converter as shown in Fig. 2(a), which is usually known as the "forward converter." Similarly, simple modification of the "buck-boost" converter results in a "flyback" converter (Fig. 2(b)) by replacing the original single inductor with a "single-ended" isolation transformer. The resulting configuration also allows the output polarity inversion of the buckboost type to be reversed as shown, where the transformer terminals of like polarity are indicated by the dots. One is therefore motivated to try a similar approach to modify the new converter topology. The obvious place to insert the 


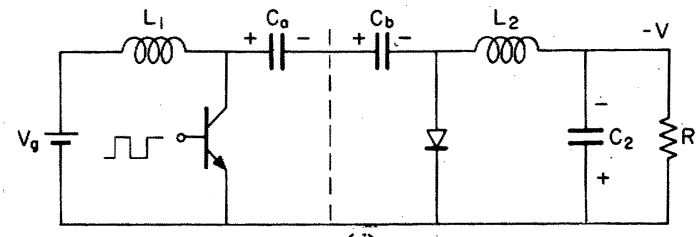

(a)

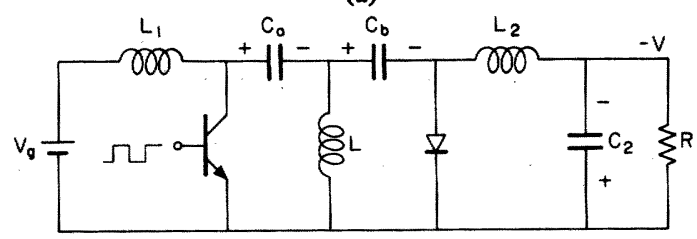

(b)

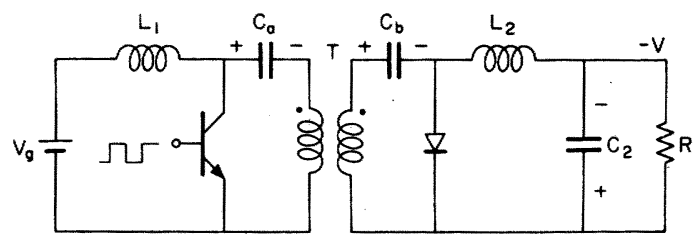

(c)

Fig. 3. Three keys steps leading to the dc-isolated version of the Cuk converter. (a) Separation of the coupling capacitance into two series capacitors. (b) Floating potential set to zero. (c) Separation of the extra inductance into two equal windings.

isolation transformer is somewhere in the inner loop containing the coupling capacitor, transistor, and diode, in which the actual energy transfer is accomplished. There are three key steps leading to a simple, elegant solution to this problem.

The first step is to separate the coupling capacitance $C$ into the two series capacitors $C_{a}$ and $C_{b}$, thus making the original symmetrical switching structure divisible into two halves, as shown in Fig. 3(a), without affecting the operation of the converter. The second step is to recognize that the connection point between these two capacitors, due to its isolation, has an indeterminate dc (average) voltage. This floating potential can then be set at zero by placing an inductance between this point and ground (Fig. 3(b)). If the new inductance is sufficiently large, it diverts a negligible current from that passing through the series capacitors, so that the converter's operation is still unaffected. The final step is merely the separation of the extra inductance into two equal transformer windings, thus providing the desired dc isolation, resulting in the basic isolated version of the new converter shown in Fig. $3(\mathrm{c})[1]$.

One of the main features of the introduction of the isolating transformer is that it has brought the least disturbance to the original converter operation. In fact, with a 1:1 transformer, the voltages and currents in the input and output circuits are the same as in the original nonisolated version. The only difference is that the original switched current loop now has evolved into two loops with equal currents circulating in the same direction.

It is instructive to consider the current paths, voltage distributions, and energy movements during the two portions of the switching cycle. Fig. 4(a) shows the conditions during the interval $D^{\prime} T_{s}$ when the switch transistor is off (open circuit). The input current, having previously stored energy in input inductor $L_{1}$, must now pass through capacitor $C_{a}$ and the

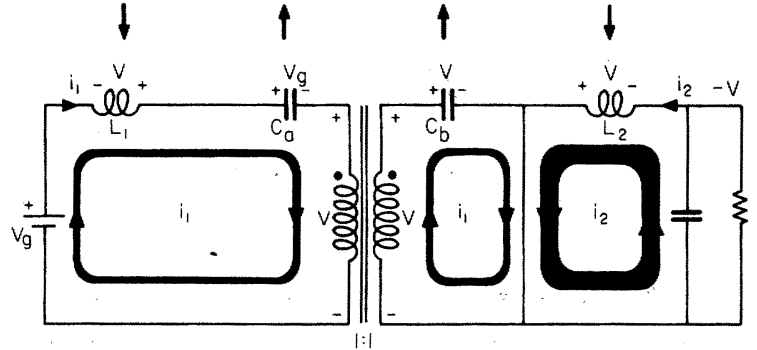

(a)

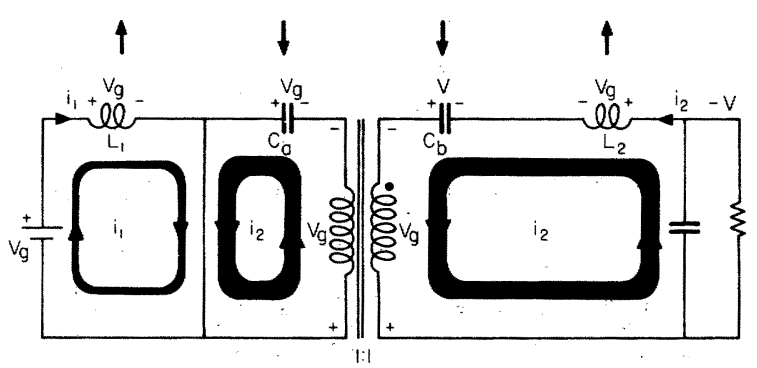

(b)

Fig. 4. Current and energy distribution in the dc-isolated Cuk converter: (a) interval $D^{\prime} T_{s}$ when the transistor switch is open; (b) interval $D T_{s}$ when the switch is closed.

transformer primary as shown. This transfers energy from $L_{1}$ (in the form of current) to $C_{a}$ (in the form of voltage, or charge). An equal reflected current in the transformer secondary charges $C_{b}$ through the now conducting diode. During this interval the output inductor $L_{2}$ is releasing its stored energy to the load as shown, and the diode thus carries the sum of both the input and output currents.

In Fig. 4(b), the transistor is turned on (closed circuit) during interval $D T_{s}$, and the input current is allowed to store energy in $L_{1}$. Since $C_{a}$ (as well as $C_{b}$ ) was charged to a positive voltage (viewed from left to right) during $D^{\prime} T_{s}$, it now discharges through the transistor and the transformer primary, transferring the stored energy to the output circuit. Note, however, that when the transistor grounds the positive side of $C_{a}$, the voltage drop across the capacitor must (instantaneously) remain the same, so that its right side is pulled down to a negative voltage. This sudden drop is passed by the transformer to the positive (left) side of $C_{b}$, and hence to the anode of the diode, cutting it off. Since the output current must now pass through $C_{b}$ and the transformer secondary, it must match the current through the primary, so that both $C_{a}$ and $C_{b}$ discharge their energy into $L_{2}$ and the load. One sees that, during this phase of the cycle, it is the transistor that carries the sum of both the input and output currents.

Some of the additional advantages of this new dc-isolated converter become apparent upon reexamining the steps which led to the inclusion of the isolation transformer. Since both windings of the transformer are dc-blocked by $C_{a}$ and $C_{b}$, there can be no $\mathrm{dc}$ in either winding. In fact, an automatic volt-second balance is achieved in the steady stage, so that there is no problem with "creep" of the transformer core operating point as can occur in push-pull isolation arrangements.

Isolation has thus been achieved in the simplest possible 


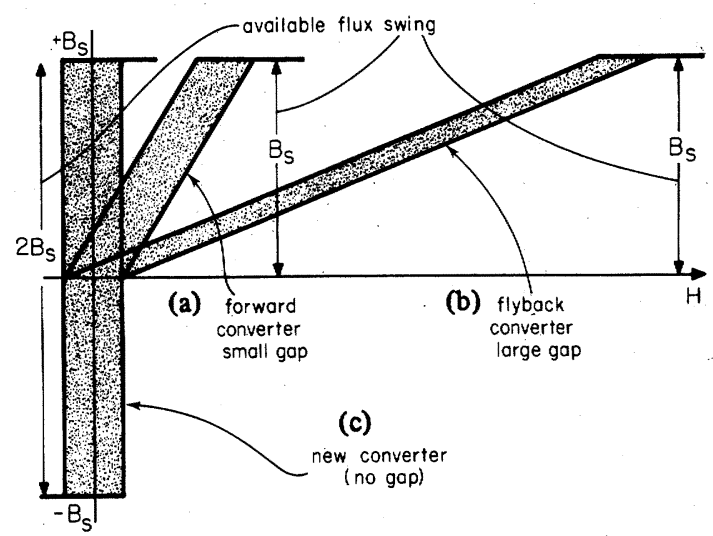

Fig. 5. Comparison of the isolation transformer utilization in the (a) forward (small gap) and (b) flyback (large gap) converters, and in the (c) new (Cuk) converter. Twice the flux swing is available in the Cuk converter.

manner by the addition of only the necessary transformer (which is single-ended) and the separation of the original coupling capacitance into two. Thus, it is apparent that an optimum-topology dc-isolated converter has been obtained which retains all the advantages of the original topology upon which it is based.

Other advantages become apparent upon comparison of the new design with the other popular isolated converters shown in Fig. 2. Specifically, for the forward converter, the core of the isolation transformer must be gapped, since the magnetizing current is available in only one direction (with an average $\mathrm{dc}$ value). The size of the core must be chosen so that the total flux excursion is no greater than the saturation flux $B_{s}$ of the core material, as shown in Fig. 5(a) [1].

In contrast, in the new converter (Fig. 5(c)), magnetizing current in the transformer is available in both directions, and so a core that is fully utilized (in terms of flux swing) in buck forward converter is only half-utilized in the new converter. Therefore, an ungapped core of half the cross section could be used, with the result that the total flux excursion would be $2 B_{s}$, and the core losses would be correspondingly halved. Similarly, the copper losses would be greatly lowered due to the reduced winding lengths. From the general point of view, these benefits all stem from the fact that in the new converter power is transmitted through the transformer during both intervals of the switching cycle, whereas the same average power has to be transmitted during only one interval in the forward converter.

Comparison of the transformer properties between the new converter and the flyback converter shows that the disparity is even more extreme, because in the flyback the core gap must be larger than in the forward converter, as illustrated in Fig. 4(b). This is because the transformer is really an inductor, and the energy transferred through the converter is stored in the magnetic field (principally in the air gap) during one part of the cycle and released during the other. Consequently, the magnetizing current, which is again available in only one direction, constitutes the total primary or secondary current instead of just a small fraction of it.

\section{THE MULTIPLE-OUTPUT EXTENSION}

Once an isolation transformer has been introduced into the new converter, several other extensions become obvious. There

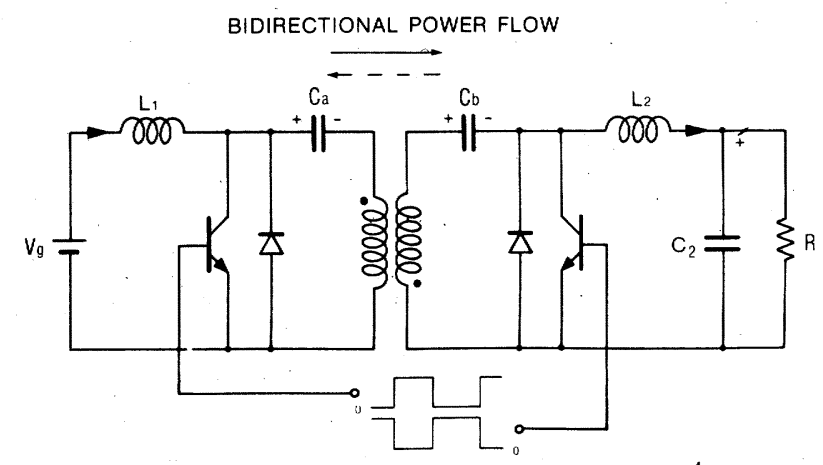

Fig. 6. DC-isolated noninverting bidirectional current Cuk switching converter, using all $n-p-n$ bipolar transistors. Compare this with Fig. 9 in Part I.

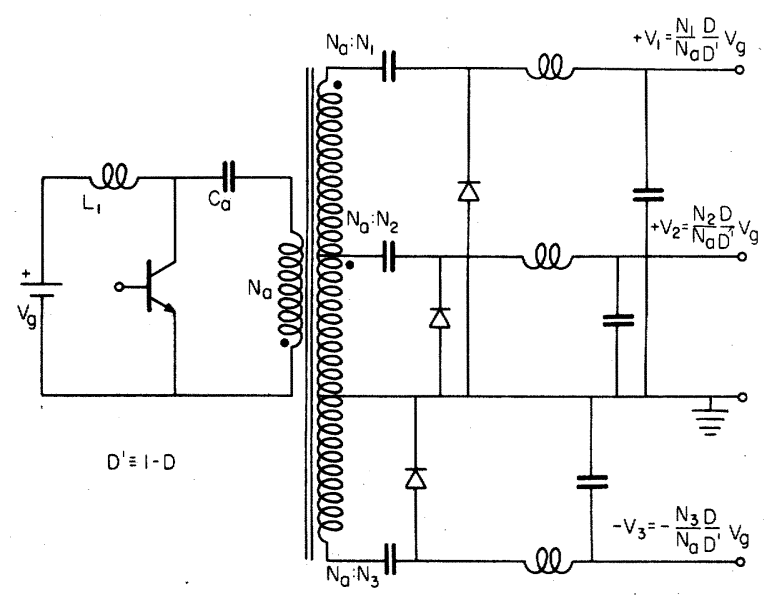

Fig. 7. Extension of the dc-isolated Cuk converter to multiple outputs with arbitrary ratios and polarities.

is no reason why the transformer should be limited to a $1: 1$ turns ratio, and therefore a turns ratio factor $N_{s} / N_{p}$ is available for either step-up or step-down in addition to gain control obtained by varying the duty ratio $D$. Thus

$$
\frac{V}{V_{g}}=\frac{N_{s}}{N_{p}} \cdot \frac{D}{1-D} .
$$

Also, as in the case of the isolated buck-boost converter in Fig. 2(b), an output voltage of the same polarity as the input voltage may be easily obtained by reversing the polarity of the isolation transformer secondary and changing the direction of the diode and polarity of the coupling capacitor on the secondary side accordingly, as shown in Fig. 6. It is interesting to note that this polarity-preserving configuration leads to an implementation of the bidirectional current (two-quadrant) version of the dc-isolated converter using all $n-p-n$ bipolar transistors, requiring only positive switch drive signals. (Compare this with the complementary pair, n-p-n and $\mathrm{p}-\mathrm{n}-\mathrm{p}$ transistor, version of the bidirectional converter presented in Part I.) The same holds for the power MOSFET implementation in which only n-channel devices are needed. This is of signficant practical importance, since $n-p-n$ transistors and n-channel MOSFET's come in all current and voltage ranges, while $p-n-p$ and p-channel devices are very limited, usually to lower current, voltage, and power ratings.

Multiple outputs of different voltages and polarities are easily obtained from multiple secondary windings, or from a tapped secondary winding as shown in Fig. 7. All of the bene- 
fits of the basic new converter topology are retained in the multiple-output versions; in particular, all the output currents and the input current are nonpulsating. When compared to the conventional approach of designing an isolated multiple output power supply using a tapped $60-\mathrm{Hz}$ transformer followed by several linear regulators, the high performance and cost effectiveness of the new method is obvious. The lower the operating frequency, the more massive a transformer must be. Instead of a bulky $60-\mathrm{Hz}$ transformer, a much smaller transformer designed to operate at the switching frequency $(20 \mathrm{kHz}$, for example) is used. Instead of the wasteful power dissipation of linear regulation, requiring heatsinks, fans, etc., the new converter typically operates at over 90-percent efficiency. An additional side benefit is that the latter design is also insensitive to the input line frequency $(50 \mathrm{~Hz}, 60 \mathrm{~Hz}, 400 \mathrm{~Hz}$, etc.), since the input voltage is supplied by rectifying the ac. Thus, by introducing $\mathrm{dc}$ isolation into the new converter, we have substantially increased its range of application.

\section{THE COUPLED-INDUCTOR DC-ISOLATED CONVERTER}

The isolation transformer was imbedded in the basic converter without altering its mode of operation, thus all the modifications and extensions applicable to the basic converter are valid in the isolated case as well. For example, the input and output inductors can be coupled on the same core as shown in Fig. 8. As discussed in Part I, zero current ripple may be obtained on either the input or the output through appropriate design of the coupled inductor.

The configuration in Fig. 8 may at first seem significantly different from the isolated converter of Fig. 3(c) apart from the coupling of the inductors. This is because the transfer capacitors $C_{a}$ and $C_{b}$ have been relocated to the other side of their respective transformer windings. This change neither alters the converter topology nor affects its conceptual operation. From the practical viewpoint, however, this alteration has important noise reduction advantages. Since the case of an electrolytic capacitor is usually common to its negative terminal, this design effectively grounds the cases of the capacitors, significantly reducing the radiation produced by the switching currents.

While magnetic coupling of the input and output inductors is very desirable for the reduction of ripple, size, and cost, it does introduce an undesirable characteristic, namely the transient reversal of output polarity on start-up. Note that in the steady state shown in Fig. 8, the average dc currents flow into the dots on the coupled inductors, contrary to the behavior of an ordinary ac transformer. During the turn-on transient, however, the ac coupling of the inductors causes the initial current pulse from the input to be reflected in the secondary in the opposite direction from the steady-state current, as shown in Fig. 9. Although the pulse is of short duration (50 $\mu$ s typically), it could result in damage to sensitive loads. The simple addition of an output clamp diode as shown limits the reverse transient to about a volt, or less if a Schottky diode is used.

Just as in the single-output case, the same opportunity for coupling exists in the multiple-output isolated converter: any or all of the inductors can be coupled on the same core, as
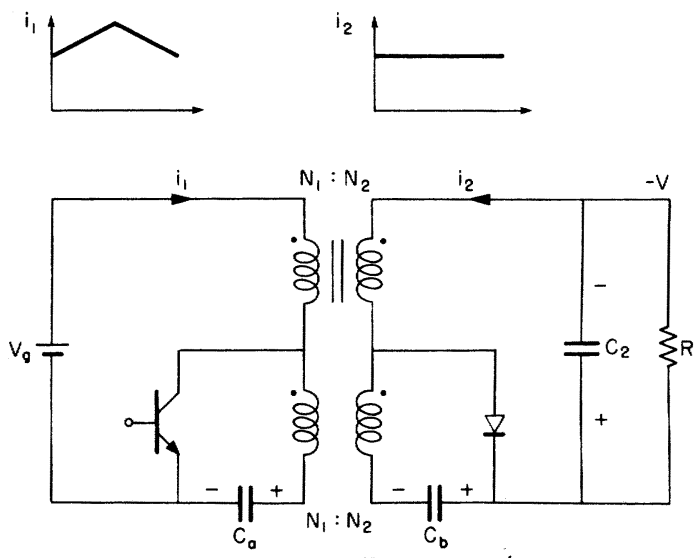

Fig. 8. Coupled-inductor dc-isolated Cuk converter.

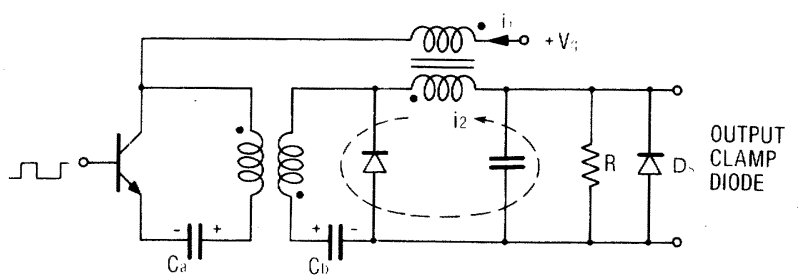

Fig. 9. Polarity reversal at start-up in a coupled-inductor Cuk converter. The output diode provides a safe path for the transient.

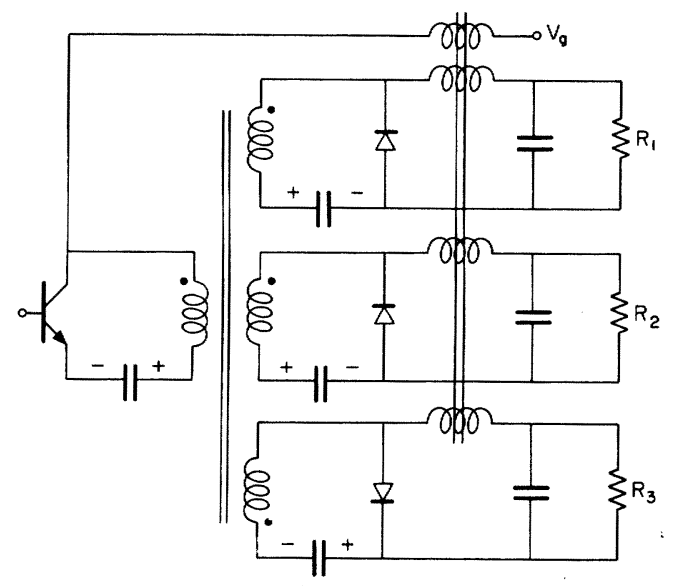

Fig. 10. Transformer-isolated Cuk converter with all inductors coupled on a single core.

shown in Fig. 10. The resulting converter has only two magnetic lumps, one for $\mathrm{dc}$ isolation and the other for input and output filtering. Again, by judicious selection of the turns ratios and coupling coefficients, the current ripple may be eliminated on the input or any of the outputs [1]

These advances notwithstanding, the possibility of zero current ripple on only one side leaves one with a feeling of incom. pleteness and a desire to accomplish this ideal goal of zero ripple on both input and output ports. That this can indeed be accomplished, with an even further reduction in the size and cost of the circuit, will once again dramatically illustrate the optimality of the new topology.

\section{THE IDEAL ZERO-RIPPLE SWITCHING DC-TO-DC CONVERTER}

In pursuit of the goal just posed, there may be many approaches to follow: for example, two coupled-inductor converters could be cascaded, with one set for zero input ripple 


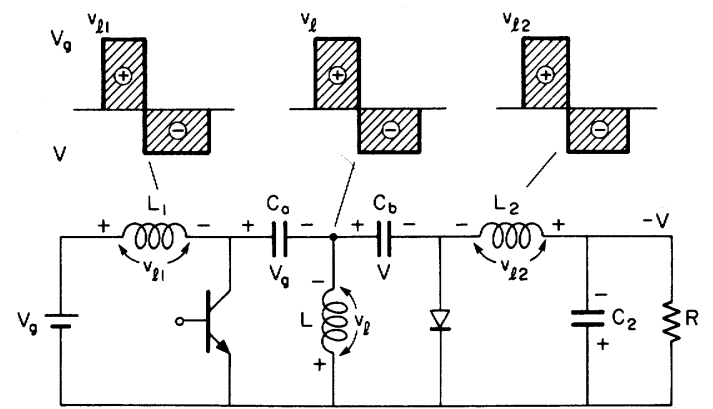

Fig. 11. Switching converter with three proportional (1:1) inductor voltage waveforms.

and the other for zero output ripple. It is quite obvious that this would be undesirable, for not only would the input power be processed twice, with nearly twice the power losses, but the parts count would be doubled and the original advantages of simplicity and reliability would be lost.

Hence the following objective is posed:

Synthesize a switching dc-to-dc converter which has the least number of components and yet leads to the ideal dc voltage and current waveforms at both input and output simultaneously.

This problem may at first seem formidable and impossible to achieve, but, based on the background material outlined so far, the coupled-inductor concept may be generalized to satisfy our goal. Recall that the original motivation for coupling the inductors was the $1: 1$ proportionality of the inductor voltage waveforms of the basic converter (Part I, Section II). The key step becomes that of finding a switching topology in which such proportional inductor voltage waveforms are abundant, hence allowing multiple application of the coupled-inductor concept. However, we have already seen such a topology in Fig. 3(b), where it was a key intermediate step toward attaining dc isolation. To emphasize the availability of the proportional switching waveforms, this configuration is shown in Fig. 11 along with the corresponding inductor voltage waveforms.

It now becomes apparent that the inner inductor $L$ may be coupled with either the input inductor $L_{1}$ for zero input current ripple or with $L_{2}$ for zero output ripple. However, with a minor modification, both current ripples may be simultaneously eliminated. By providing two inductors $L_{a}$ and $L_{b}$ in parallel instead of the single $L$ and coupling them as shown in Fig. 12(a), both ripple currents may be transferred inside the converter, resulting in pure dc at each port.

The two inductances $L_{a}$ and $L_{b}$ are not coupled magnetically, but are only connected in parallel electrically, hence the configuration has two magnetic lumps as shown in Fig. 12(b), in which gapped "U-cores" are used as one possible implementation. However, these two lumps could be even further reduced by coupling the windings $L_{a}$ and $L_{b}$ magnetically as well. This is equivalent to magnetically coupling the single inner winding of Fig. 11 to both input and output inductors simultaneously as shown in Fig. 13. Note that, as in Fig. 12, the input and output inductors $L_{1}$ and $L_{2}$ are not directly coupled as indicated by the two separate core lines for the input and output sides. However, unlike the configuration in Fig. 12, the

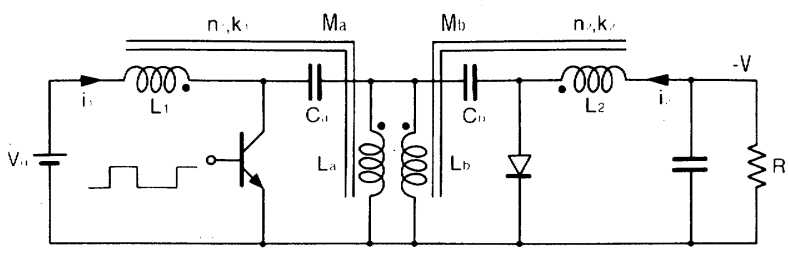

(a)

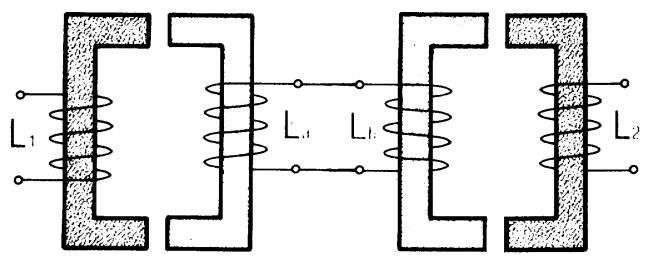

(b)

Fig. 12. (a) Converter with zero current ripple at both input and output, and (b) the realization of the magnetic circuits.

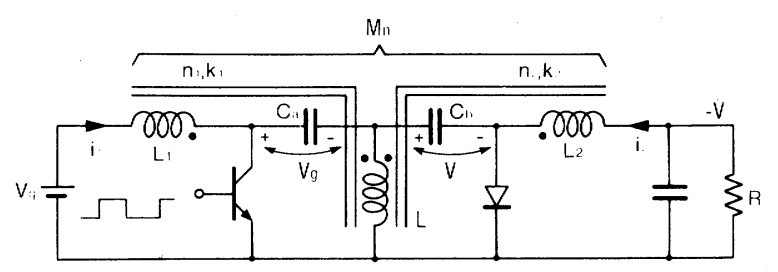

(a)

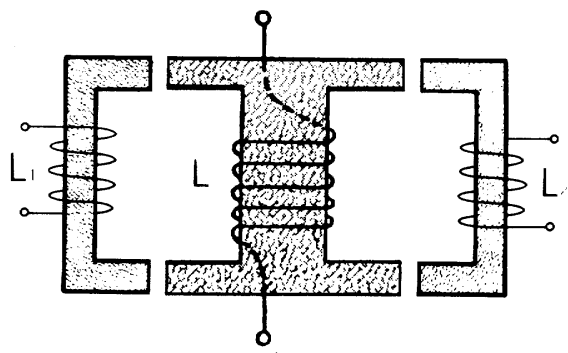

(b)

Fig. 13. Zero-ripple switching converter with (a) shared inner inductor and (b) one possible core implementation.

adjustments for zero ripple are no longer independent, due to the magnetic coupling through the common core $(L)$. Changing one air gap alters the ripple charactierstics of both sides. Nevertheless, the adjustments are highly convergent and the ideal zero-ripple case is readily obtained [2].

None of the switching topologies shown so far in this section has the important dc-isolation property. However, this feature is now easily obtained by replacing the single winding of the inner leg of the magnetic circuit $(L)$ with separate, isolated windings for the input and output, as shown in Fig. 14, with no additional magnetic material required. This step is completely analogous to the one described earlier which led to the original dc-isolated version of the converter (Fig. 3(b)(c)). However, in the single magnetic circuit of Fig. 14, additional benefits are obtained.

The added isolation transformer now has a double role-it not only provides the isolation, but also, through magnetic coupling with the input and output inductors, results in ideal dc current at both input and output simultaneously.

From the magnetic circuit realization in Fig. 14(b), it may seem that a rather special magnetic core configuration is needed, one comprising more than a single core structure. 


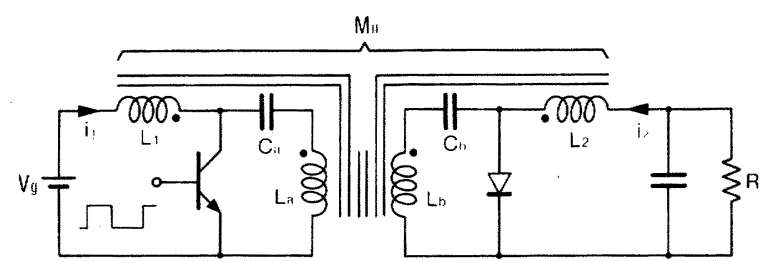

(a)
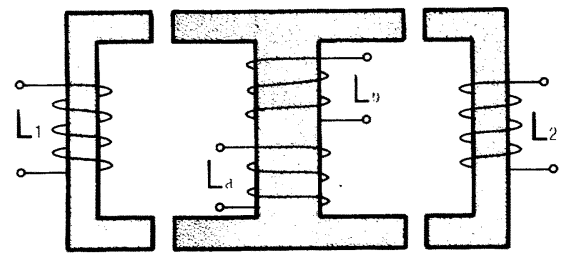

(b)

Fig. 14. (a) DC-isolated version of the zero-ripple converter obtained by adding a single winding to the magnetic circuit of Fig. 13(b).

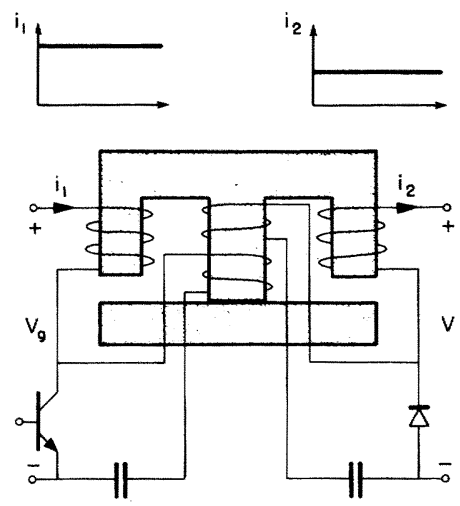

Fig. 15. The simplest realization of an ideal dc-to-dc transformer: a four-winding magnetic circuit, a transistor and diode, and two capacitors.

However, the structure illustrated was used only for conceptual reasons to clearly explain each step of the development. A number of widely used standard magnetic cores, such as common $E-I$ cores, for example, can be used for the practical implementation of the switching converter in Fig. 14(a) and its single magnetic circuit. In Fig. 15 the $E-I$ core implementation of the integrated inductor-transformer differs from standard cores only in that each of its outer legs contains an air gap, as opposed to the usual case in which only the inner leg is gapped. The fact that three bobbins are used (one winding on each leg) is not unusual and is, in fact, common in threephase transformer designs using $E-I$ cores.

This resemblance in core structure is not a mere coincidence. Merging the three single-phase transformers into a single three-phase transformer results in corresponding savings in core material due to the merging of the magnetic fluxes and the sharing of the common flux paths. Similarly, the core configuration of Fig. 15, by merging the two separate inductors $L_{1}$ and $L_{2}$ and the isolation transformer into this single magnetic structure, results in substantial savings in size, weight, and efficiency.

Thus, a truly optimum dc converter is obtained which has both outstanding features: true dc currents at either port, as well as dc isolation, in the simplest possible topology consisting of a single magnetic circuit, a transistor and diode, and two capacitors. Under the control of a clock signal with

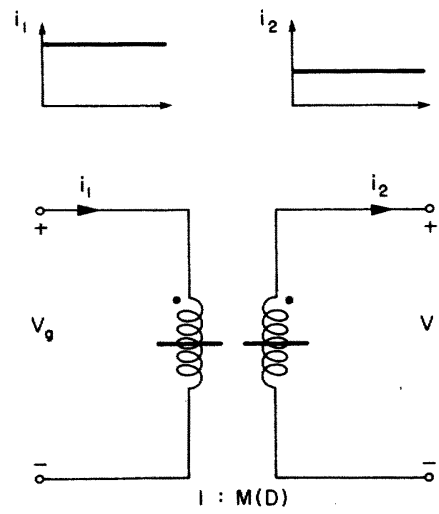

Fig. 16. Equivalent circuit symbol denoting the ideal dc-to-dc transformer with constant input and output currents, used as a model of the converter in Fig. 15.

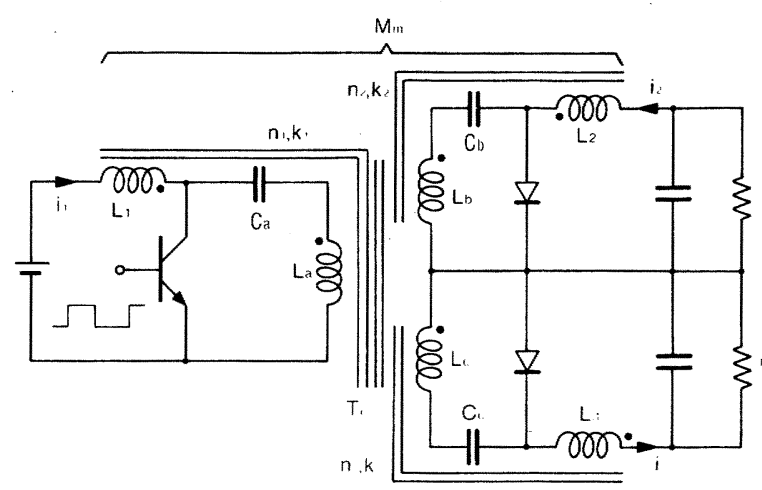

(a)

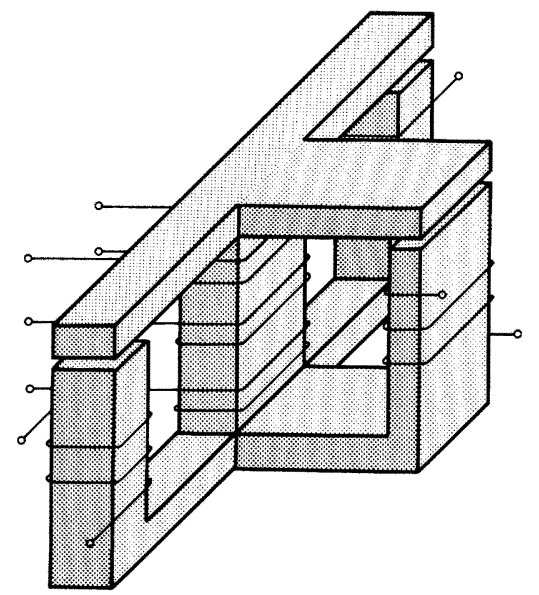

(b)

Fig. 17. Multiple-output converter with zero current ripple on (a) the input and both outputs and (b) the single magnetic circuit implementation.

variable duty cycle $D$, the converter becomes the physical realization of a dc-to-dc "transformer" with an electronically variable "turns-ratio" (Fig. 16).

A natural extension of this zero-ripple converter configuration (Fig. 15) to multiple outputs is shown in Fig. 17(a). There, a single magnetic circuit $M_{m}$ with six windings appropriately coupled is capable of providing zero current ripple not only at the input but simultaneously at both outputs as well. The actual physical implementation of its magnetic circuit is a simple three-dimensional extension of the one-output core configuration, as illustrated in Fig. 17(b), providing an ad- 
ditional flux loop common to the central core but separate from the other legs. The three windings of the isolation transformer are placed on the central leg, while the input and two output windings are placed on the three outer legs. Interestingly enough, such a configuration has recently been proposed for a different application, the "Variable Leakage Transformer," in which the third leg is used by a control winding [3]. The extension of this zero-ripple configuration to provide additional outputs (three or more) is performed similarly.

\section{THE CONCEPT OF INTEGRATED MAGNETIC CIRCUITS}

Apart from the usefulness and practical advantages of the various zero-ripple converter configurations previously outlined their significance extends even futher, since their development has led to a new and general integrated magnetic circuit concept.

The coupled-inductor extension of the new converter was the crucial first step in that direction. There, from the conceptual viewpoint, two magnetic components (inductors), which until then had been used only separately to perform their function in circuits, were for the first time integrated into a single magnetic circuit (single core) with two windings. While it looked like a classical ac transformer from the structural viewpoint, it indeed performed the function of two separate inductors, when polarity marks and actual current directions were taken into account. Furthermore, it even outperformed the separate inductors by significantly reducing the size, weight, and losses.

The natural outgrowth, and in fact the generalization, of this concept is achieved when such diverse magnetic components as inductors and transformers, again used exclusively as separate elements until now, are merged into an integral magnetic circuit with multiple windings. When put into the proper circuit configurations (such as those of Figs. 13-15, 17), they not only perform the original functions, but even tremendously improve the performance of the converter (ripple currents reduced to zero), with additional savings in size and weight and increased efficiency.

An analogy with the development from discrete electric circuits to modern integrated electronics can also be drawn. Just as electronic components and their interconnections were integrated on a common silicon medium, with electron current as the binding carrier, we now have diverse magnetic components integrated into a single magnetic circuit, with the core material as the medium and magnetic flux as the binding carrier. Whereas previously we could describe the current distribution in a complex electronic circuit, we now, in the case of switching converters, have a complex magnetic circuit, with a corresponding flux distribution, which is superimposed on and related to its electrical complement. For this process of reducing the separate magnetic components into a single magnetic circuit with accompanying savings in size and weight, and owing to its similarity with the integration of electrical circuits, a generic name integrated magnetics is proposed.

This new concept is applicable to a broad spectrum of electrical circuits involving magnetic components. To illustrate this, we will demonstrate its use in different converter configurations, with similar benefits. When applied to the SEPIC con-

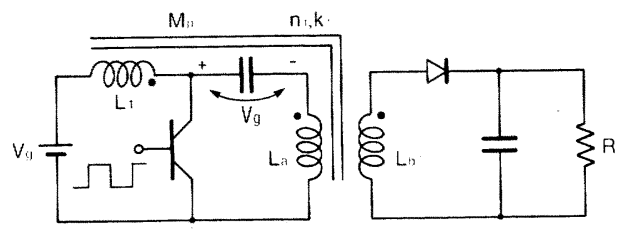

(a)

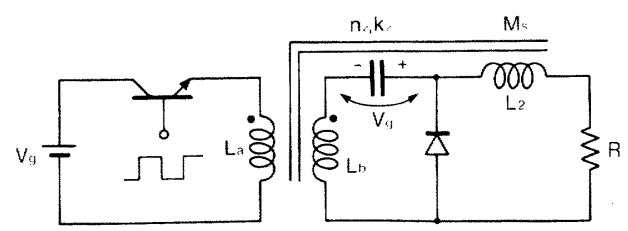

(b)

Fig. 18. The integrated magnetic circuit concept, when applied to two converter configurations, (a) the SEPIC converter and (b) its dual counterpart result in zero current ripple at the (a) input or (b) output.

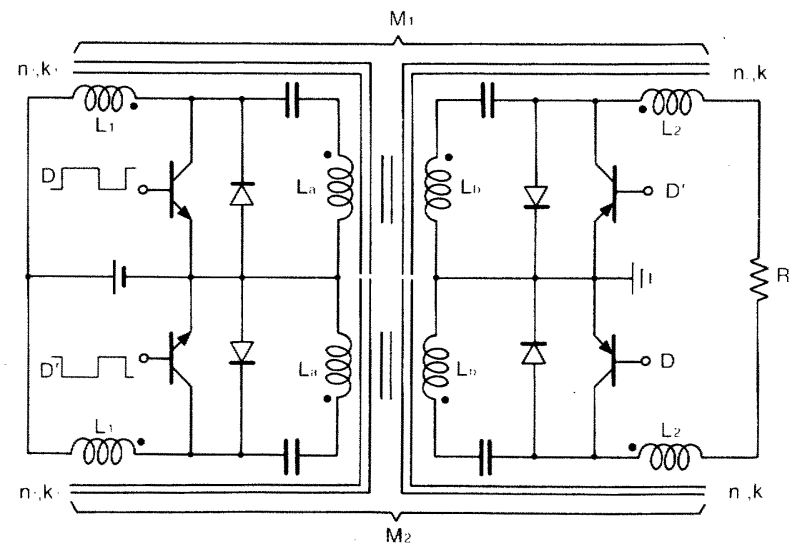

Fig. 19. Integrated magnetics applied to the Cuk switching power amplifier. Compare with Part I, Fig. 19.

verter [4], shown in Fig. 18(a) or its dual counterpart (b), it results in zero-ripple current on either the input (a) or output (b) side.

Also, of course, the magnetic elements used in the switching power amplifier based on the new converter (described in Part I) may be similarly integrated. In the configuration shown in Fig. 19, the basic amplifier has been modified by employing integrated magnetics to have dc isolation (for use with ac line voltage input, for example) and zero ripple on both the input and the load. The resulting circuit has only two magnetic circuits, each with four windings.

These examples illustrate how, in a complex switching configuration consisting of a number of storage elements (inductors, transformers, and capacitors) and switches, interconnected to perform some useful function, such as dc-to-dc conversion, dc-to-ac inversion, the otherwise separate magnetic components can be merged into a integral magnetic circuit with multiple windings. The prerequisite for such a simplification is the existence of synchronized and proportional voltage waveforms on the inductors and transformers, as in the case of dc-isolated version of the new converter previously described.

\section{CONCLUSIONS}

A long and most rewarding exploration of switching dc-todc converter topologies has come to its fruition in establishing a practical switching configuration which truly emulates 


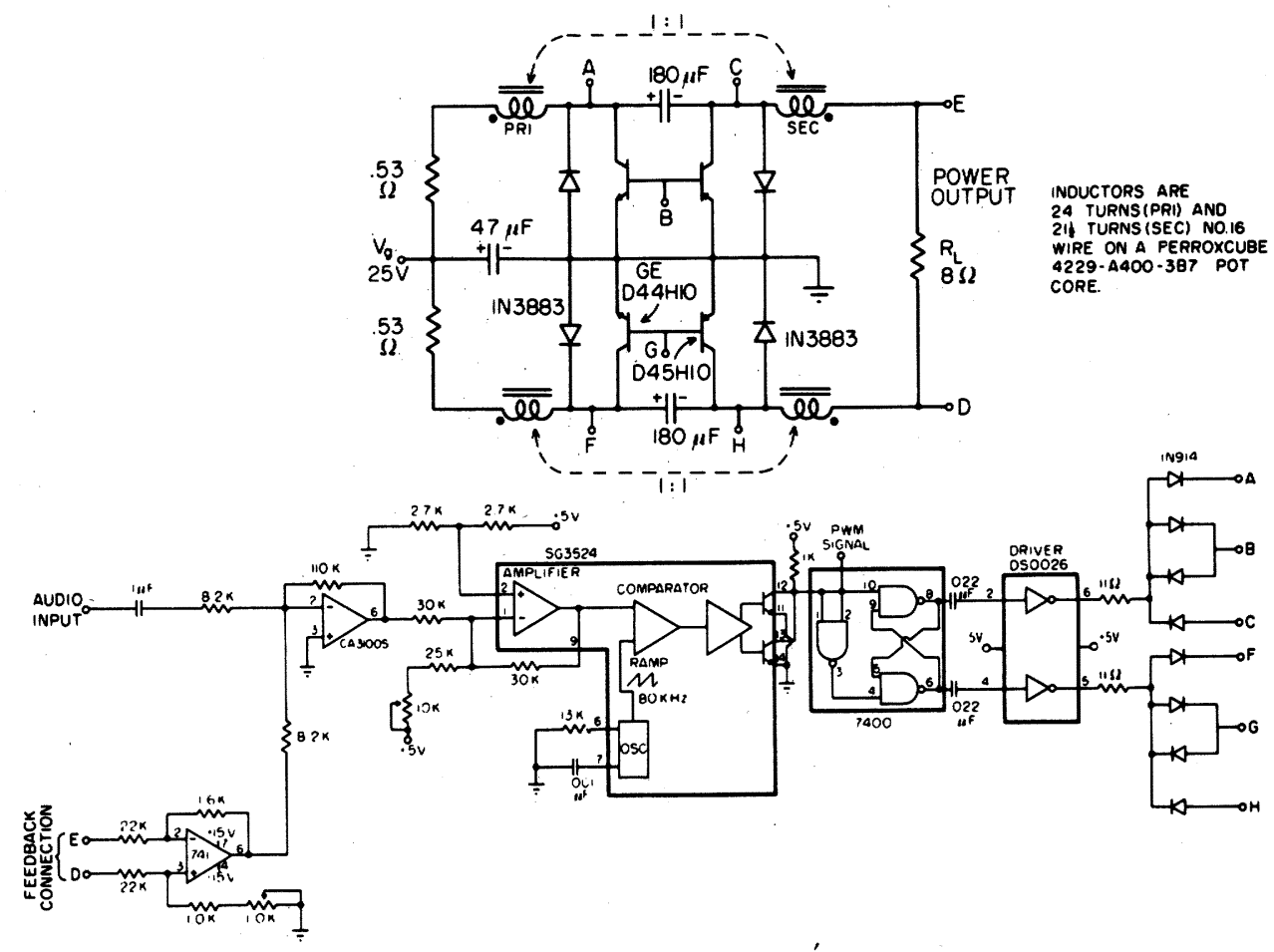

Fig. 20. A push-pull switching power amplifier based on the Cuk coupled-inductor power stage.

the ideal dc-to-dc transformer function. Let us conclude by summarizing some of the major milestones encountered along the way.

The traditional approach taken by many researchers and practicing engineers is indeed very simple: let us first get the required dc-to-dc conversion function by using some simple switching mechanism (such as buck or buck-boost) and then cure its problems (such as pulsating input or output currents and severe EMI) later, such as by adding complex filter and storage elements to "clean up" the signals.

Our approach, however, has been just the opposite: from the very beginning we considered that storage and energy transfer elements, together with the switches, are an integral part of the problem. Moreover, we have not only attached extreme importance to converter topologies but now have extended the previous topology of electrical connections into its complementary topology of magnetic connections through the newly introduced integrated magnetics approach.

At every point in the development, we introduced additional complexity by adding circuit elements, electrical or magnetic connections for only two reasons: because it was the optimal method of obtaining a desired feature or function-or as an intermediate step towards a further simplification. Even when a seemingly simple configuration was achieved in the basic converter, the desire to still further simplify and improve performance led to the new coupled-inductor concept, which has now been generalized into the integrated magnetics concept.

As a result of this approach, dc-to-dc conversion topologies have now been extended to include bidirectional power flow (two-quadrant converters) and dc-to-ac inversion and power amplification (four-quadrant converters) as presented in Part $\mathrm{I}$, and now to the dc-isolated and ideal zero-ripple converters presented here. Thus, the approach has been generalized to include the whole spectrum of Power Electronics applications.

\section{APPENDIX A CIRCUIT SPECIFICATIONS FOR A SWITCHED-MODE POWER AMPLIFIER}

In Part I of this article the application of the Cuk power stage to a new push-pull power amplifier configuration was presented, along with a detailed discussion of its performance. The Cuk amplifier features broad frequency response (flat over $20 \mathrm{~Hz}-20 \mathrm{kHz}$ ), low distortion (less than 0.1 percent THD), and high efficiency (approximately 90 percent), using inexpensive, commonly available components. Its capability for bidirectional power flow, as with the other bidirectional configurations described in Part I, make it highly suitable for use in driving loads from which stored energy can be recovered, such as for performing regenerative braking of $\mathrm{dc}$ motors.

Interestingly enough, the same amplifier may be used for the servo control of ac induction (Tesla) motors. When an ac signal source at a constant slip frequency relative to the motor's speed is used as input, optimum torque control, as well as the desirable regenerative braking, may be obtained.

We present here the design of a working Cuk amplifier suitable for use either as an audio amplifier or a servo driver for small dc motors (up to $40 \mathrm{~W}$ ). The circuit, shown in Fig. 20 , is based on the coupled-inductor, nonisolated version of the Cuk converter [5]. Note that the load is connected differentially across the two power stages, instead of relative to ground. (A photograph of the assembled amplifier was shown at the end of Part I.) Each of the coupled inductors uses a single ferrite "pot" core assembly, wound as specified on the single bobbin, secondary first. Observe the polarity markings on the diagram so that current flowing "into the dot" on each winding will travel in the same direction around the core.

The voltage across the load $(E-D)$ is differentially sensed by the 741 op amp and compared with the input signal using a RCA CA3100S op amp. $R_{1}$ is adjusted to obtain the best 


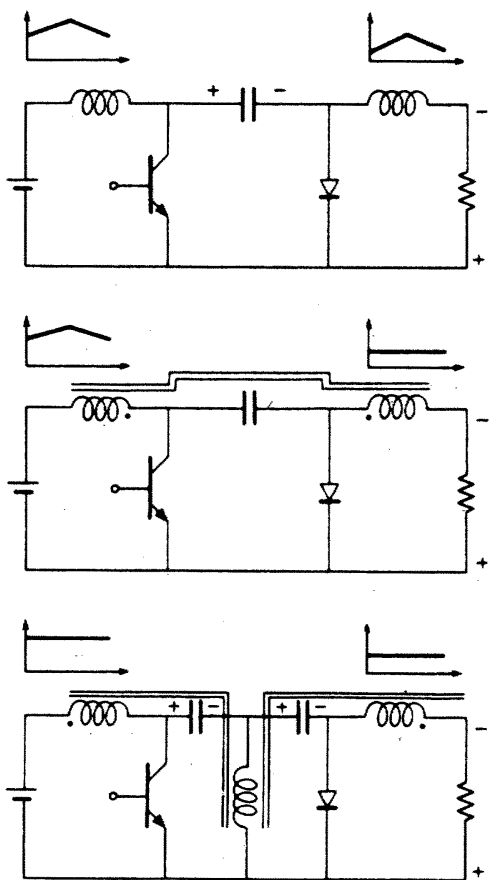

(a)
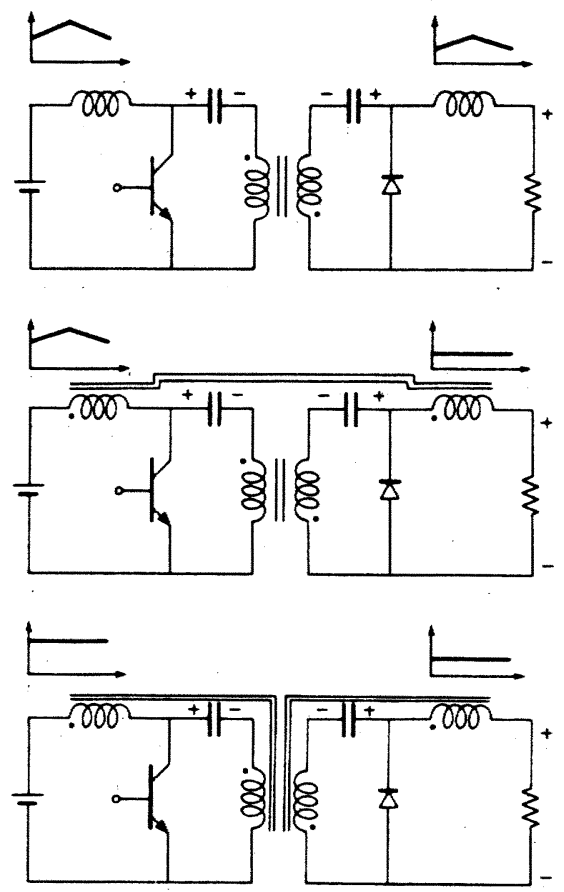

(b)

Fig. 21. Basic Cuk converter. (a) Nonisolated version and (b) isolated version.

differential output from the 741 . The excellent frequency and phase response of the Cuk power stage eliminates the need for any frequency compensation of the feedback. A switching regulator integrated circuit SG3524, by Silicon General, is used in the feedback loop to generate a pulse-width-modulated (PWM) signal with an $80-\mathrm{kHz}$ switching frequency. $R_{2}$ provides dc balance and is adjusted to produce zero output voltage in the absence of an input signal. Logic gates $(7400$ NAND) connected as a flip-flop produce two out-of-phase input signals for the DS0026drivers, which produce the $\pm 5-\mathrm{V}$ drive signals for the switching transistors. Diodes (1N914) were used in a modification of the "Baker clamp" to improve the transistor switching times, while still retaining the feature of automatically preventing the overlap of the transistor on times.

In many dc motor control applications, it is desirable for the servo input voltage to determine the current to be supplied to the motor instead of the voltage. Current sensing for use in feedback control can be accomplished by inserting a small ( 0.1 $\Omega$ ) sense resistor in series with the load and applying the voltage across it to the feedback inputs $E$ and $D$, with the gain of the differential amplifier suitably increased.

The $0.53-\Omega$ resistors on the input side of the switching power stage serve to optimize the harmonic distortion characteristics of the amplifier at a slight expense in operating efficiency (see Part I). For applications in which distortion is not a critical factor, these resistors may be omitted.

\section{APPENDIX B \\ THE EVOLUTION OF ZERO-RIPPLE DC-TO-DC SWITCHING CONVERTERS}

The search for new efficient, low-noise switching converter topologies was originally motivated by the desire to reduce or possibly eliminate some of the major problems which limit "switchers," such as the large pulse currents at either the input or the output port or even both. For example, in a conventional buck-boost (flyback) converter, both input- and outputport currents are large pulses, leading to severe conducted and radiated EMI. The basic Ćuk converter (nonisolated version) was a substantial step in the direction of reducing this problem by creating nonpulsating input and output currents. However, the unique topology of the Cuk switching converter and in particular the proportional voltage waveforms on their two inductors has led to powerful new concept: the coupling of inductors. Besides the obvious reduction in complexity, yet another significant advancement has been acomplished:current ripple at either input or output is not only reduced but completely eliminated. The natural outgrowth of this new technique is a switching configuration which employs a single magnetic circuit with three windings and achieves zero current ripple at both ports simultaneously.

For many practical applications, dc isolation between source and load is essential. The next key step in the development is the incorporation of an isolation transformer in an optimum single-ended manner, which leaves the fundamental features of the basic Cuk converter intact. Hence, coupling of the input and output inductors leads again to zero current ripple at either end. Finally, the crowning achievement of the converter development is its final evolution into a topology which truly emulates the desired electronically controllable ideal dc-to-dc transformer. This ultimate switching dc-to-dc converter configuration possesses both outstanding features: true dc currents at both ports, and dc isolation in the simplest possible topology, consisting of a single magnetic circuit with four windings, two capacitors, and a single switch implemented by the usual transistor-diode combination. (See Fig. 21.)

In summary, a long journey in the development of opti- 
mum switching converter topologies has been successfully completed: from the conventional buck-boost with large pulsating currents at both input and output ports, through new converters with both currents nonpulsating, to an ideal dc-to-dc switching converter, all featuring nonisolated as well as isolated versions. The basic Ćuk converter, as well as many of its extensions and applications, are protected by a series of patents [7]-[10].

\section{REFERENCES}

[1] R. D. Middlebrook and S. Cuk, "Isolation and multiple output extensions of a new optimum topology switching dc-to-dc converter," in Proc. 1978 IEEE Power Elec. Specialists Conf.
[2] S. Ćuk, "A new zero-ripple switching dc-to-dc converter and integrated magnetics," in Proc. IEEE Power Elec. Specialists Conf., May 1980

[3] H. Hirayama, "Simplifying switched mode converter design with a new variable leakage transformer topology," in Proc. 7th Nat. Solid-State Power Conversion Conf., Mar. 1980.

[4] R. Massey and E. Snyder, "High-voltage single-ended dc-dc converter," presented at IEEE Power Elec. Specialists Conf., 1977.

[5] S. Cuk and R. Erickson, "A conceptually new high-frequency switched-mode power amplifier technique eliminates current ripple," in Proc. 5th Nat. Solid-State Power Conversion Conf., May 1978.

[6] L. Rensink, A. Brown, S.-P. Hsu, and S. Ćuk, "Design of a kilowatt off-line switcher using a Ćuk converter," in Proc. 6 th Nat. Solid-State Power Conversion Conf.

\title{
A Complementary Current Impulse Commutated Thyristor Inverter
}

\author{
PHOIVOS D. ZIOGAS, MEMBER, IEEE
}

\begin{abstract}
A complementary current impulse commutated thyristor inverter is discussed in this paper. In this configuration, the two pairs of diagonally opposite located thyristors, which comprise a phase inverter, are alternately utilized as commutation and as main thyristors. Therefore, simultaneous conduction of two complementary thyristors becomes the normal means of achieving commutation, and the problem of "shoot-through" across the dc source is eliminated.

The above feature coupled with an extremely simple thyristor firing scheme results in a highly reliable inverter circuit.
\end{abstract}

\section{INTRODUCTION}

$\mathbf{F}$ IG. 1 DEPICTS a single-phase full-bridge circuit module of the most used configuration of voltage source thyristor inverters. This circuit, known as the McMurray-Bedford (MB) inverter [2], utilizes current impulse commutation and employs a small number of lightweight energy efficient components that allow high commutation frequency rates. In particular, thyristor pairs $T_{1,4}$ and $T_{2,3}$ are alternately employed to modulate the output voltage and load current, while thyristors $T_{1,2,3,4}^{\prime}$ are employed to commutate their respective main thyristors. In order to ensure commutation, this circuit requires a starting gating sequence that must be repeated after each fault-clearing operation. The circuit (Fig. 1) is also predisposed to current "shoot-throughs" that may result from commutation failure of main or auxiliary thyristors.

Fig. 2 depicts a single-phase full-bridge circuit module of the proposed inverter configuration. This circuit utilizes the temporary isolation of the inverter from the dc source, pro-

Manuscript received August 5, 1980; revised August 13, 1982.

The author is with the Department of Electrical Engineering, Concordia University, Montreal, Quebec, Canada.

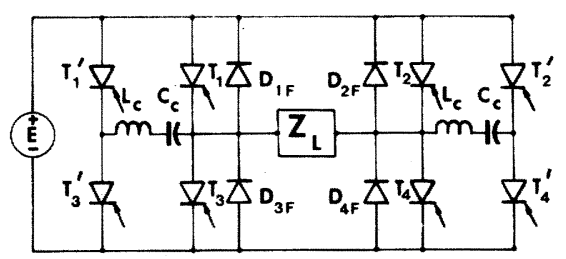

Fig. 1. A single phase full-bridge leg of the McMurray Inverter.

vided by reactor $L_{0}$, to perform current commutation. Specifically, a conducting pair of thyristors can be turned off by turning on the nonconducting pair. Consequently, in order to turn off thyristors $T_{1}$ and $T_{4}$, one must turn on thyristors $T_{2}$ and $T_{3}$.

The main advantage of the proposed inverter circuit (and commutation scheme), as compared to the existing ones (that employ impulse current commutation), is a greatly improved reliability. The reasons are: a) the problem of simultaneous conduction of any two complementary thyristors has been eliminated; b) load faults can be easily cleared because of the isolating (circuit from dc source) action of reactor $L_{0}$; and c) the thyristor firing sequence is the simplest and safest possible.

The disadvantages of the proposed inverter circuit are a) higher circuit losses, and b) lack of a zero-volts interval across the load.

\section{CIRCUIT ANALYSIS AND DESIGN}

The objective of this section is to discuss and analyze the performance of the proposed inverter circuit (Fig. 2). The analysis yields a set of design equations that specify the size 